\title{
A Virtual Switching Model for Improvemnent of Call Quality in Integrated Communication System
}

\author{
Amanze, Ruth C., Okolie, S.O., Kuyoro S.O, Alao , O.D
}

\begin{abstract}
Poor voice quality in VoIP models during communication has been a common occurrence which VoIP users experience, this can be frustrating when users cannot communicate efficiently. Most people find it difficult to think straight when they make calls and there is an echo. In addition to this frustration, the caller's money, time, effort, energy is all wasted without compensation of any kind. Users are also frustrated by not receiving, transmitting or misunderstanding voice messages correctly. Given the need for voice quality in calls, it is of no importance when there is no proper communication. This study aims to reduce the threat of bad calls and improve the quality of voice calls. Nonetheless, we need to raise the filter duration to a high value in some real telecom's environments with long echo delays. But, because of high computational complexity, it is not efficient in efficiency. In this study, we suggest a solution that uses a computational formula to compensate long echo, delay, packet loss, jitter and noise. The model designed was developed using MATLAB 2019b. This approach demonstrated productivity in terms of both voice quality and system speed.
\end{abstract}

Keywords-Communication; Call Quality; Model; Switching and Voice.

\section{I.INTRODUCTION}

VoIP was one of the ways in which the cost of calling over long distances was reduced, particularly for international calls. Also, the penetration rate of mobile broadband is driving the shift to Internet Protocol (IP) services. Phones have grown to be multidimensional devices that can be used as other avenues of communicating and not just devices for receiving and making calls. However, the issue of voice clarity in communication, when receiving or making calls, has remained a challenge and a concern to phone users. Call quality is affected by challenges like echo, jitter, noise, packet loss and delay[1]. Call quality can be divided into three: listening qualities, conversational characteristics and transmission characteristics. Quality of hearing is related to quality of sound, conversational quality refers to both conversation and listening qualities which may include echo or delay issues that can affect conversation, whereas, transmission qualities include network connection that is used to transmit the voice,

Revised Manuscript Received on May 05, 2020.

* Correspondence Author

Amanze, Ruth C*., Department, School of Computing and Engineering Sciences Babcock University.

Okolie, S.O., Department, School of Computing and Engineering Sciences Babcock University.

Kuyoro S.O, Department, School of Computing and Engineering Sciences Babcock University.

Alao, O.D, Department, School of Computing and Engineering Sciences Babcock University.

(C) The Authors. Published by Blue Eyes Intelligence Engineering and Sciences Publication (BEIESP). This is an open access article under the CC BY-NC-ND license (http://creativecommons.org/licenses/by-nc-nd/4.0/) in the area of network service quality. According to [2] mobile devices have gained $94 \%$ penetration in Nigeria. With this high level of penetration, there is the need to solve the problem of call quality for users to enjoy the utilities provided by network operators. With increasing globalization, communication has become a medium of empowerment because information is power. According to [2-3] Nigeria's current population as at Thursday, February 21 , is about $199,108,848$. Due to this large population in Nigeria, getting-in-touch with one another will be difficult using the face to face method, hence the need for mobile communication. Various social media have been used in order to accomplish the quest to communicate with one another. The quality of voice calls over the Internet is extremely important for customers of integrated communication technologies, and therefore it is vital to examine the techniques to enhance it. Coding/Decoding (Codecs) have been established in literature as the only way for payload reduction which unfortunately results in the reduction of voice quality.

\section{II.REVIEW OF RELATED WORKS}

[4] reviewed various scholarly articles to have a comprehensive and concise report on VoIP. Their opinions include the conceptualization of the Next Generation (NGN), a packet-based network capable of delivering telecommunication services, although VoIP may make use of existing structures. The features of service are dependent on the underlying transport technology and can also make use of various broadband as well as QoS enabled transport systems. IP multimedia (IMS) Subsystems are the common architecture in which NGN operates and works on all networks using packet switching, whether fixed or wireless. This paper did not give a precursor in the next generation network (NGN) before its conclusion and was only mentioned in vaguely.

[5] researched the effect of the codec and QoS technique used on speech transmission quality in IP networks. Ten samples were measured for the various methods of controlling and preventing congestion. The intervals of confidence were also calculated by a mathematical technique. In comparison to high-bit rate codecs, low-bit rate codecs yielded worse outcomes, however low-bitrates could be helpful in low bandwidth. In this job, the techniques were not addressed only the outcomes were shown graphically. [4-5] The objective of carrying out this research was to evaluate the voice quality in Long Tern Evolution (LTE) through the use of various codec mode set. This study has shown that all mode sets worked in the same manner under distinct circumstances of track loss.

Published By:

Blue Eyes Intelligence Engineering

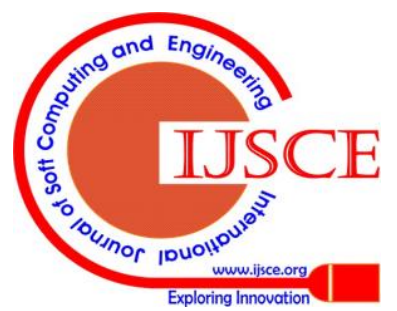




\section{A Virtual Switching Model for Improvemnent of Call Quality in Integrated Communication System}

It was found that the MOS readings were significantly different for different mode-sets and that the highest modeset experienced the highest call quality. Call quality was consistent to a certain point when the device reached the edge of cell after which a drop in audio quality was experienced. Despite this, none of the codec mode-sets showed a distinct advantage over the other. It was also found that the controlled test and field test showed a marked difference in Downlink (DL) Path loss greater than 130dBIn field testing, the locking function of physical cell identity (PCI) was also used but this was not recorded during controlled test, which equals data collection was not very optimized for the study.

\section{III.3.0 METHODOLOGY}

The average time per message in a virtual Switching Circuit expressed as

$$
T=\sum_{i=1}^{M} \frac{\lambda_{i}}{\gamma} K_{i}
$$

Where $\mathrm{M}$ is the amount of network channels,

$\lambda_{i}$ the total traffic on the ith channels,

$\gamma$ is the cumulative outside network traffic and

$K_{i}$ is the average time per $i t h$ channel message

Equation (1) can be reprogrammed as a balanced network

$T=n K_{i}$

where

$n=\sum_{i=1}^{M} \frac{\lambda_{i}}{\gamma}$

In equation (3)n is the average number of storage nodes reached by a message from its source node. It relies on routing approach, network topology. A tandem network of $n$ nodes can be formed for evaluation when recognized. The threshold value rules formulation of non-noisy channels are as follows:

(a) where the service requirement is lower or equal to the threshold value of an externally arriving message

(b) the message is not broken into packets and is passed on as such to the network. (as shown in Equation (4)

(c) If a service requirement is higher than the threshold value of the message that arrives externally, the message will be segmented in narrower packets with maximum moment before mobility across the network begins. If an exponential distribution follows the service times of noisy channel for the VoIP with an average of one message, then the following relationships can be shown as real:

$$
\begin{aligned}
& p(A)=P\left[x: x \leq X_{t h}\right]=1-\exp \left(-\mu X_{t h}\right) \\
& p(B)=P\left[x: x \leq X_{t h}\right]=\exp \left(-\mu X_{t h}\right)
\end{aligned}
$$

Where:

$p(A)$

$p(B)$ is the transmitted voice signal

$P(B)$ is the received voice signal

$X_{\text {th }}$ is the threshold constant

$\mu$ is the mean packet sent/received

Thus $E[N]=$ Expected packet number per message (illustrated in (8)Then

$$
E[N \mid A]=1 \quad \text { (6) no segmentation }
$$

Where $\mathrm{D}$ is greater than the smallest

integer $X_{t h} / l_{p} E[N]$

Where $l_{p} \quad$ is the length of the transmitted or received packet

$$
E[N \mid B]=\frac{p(B) D+p(B)}{p(A)}+D
$$

(8)

For receiving packets, this condition holds

Threshold constant $=$ length of packets

$$
X_{t h}=l_{p}
$$

Substitute 3.4, 3.5 into 3.8

$$
E[N \mid B]=\frac{\exp \left(-\mu D l_{p}\right)+p(B)}{1+\exp \mu l_{p}}+D
$$

(3.8) (Source : Authors work)

$$
E[N \mid B]=D+\frac{\exp [-\mu]\left(D l_{p}-X_{t h}\right)}{\left[1-\exp \left(-\mu l_{p}\right)\right]}
$$

$$
E[N]=E[N \mid A] P(A)+E[N \mid B] P(B)
$$

Or

$$
E[N]=1+(D-1) \exp \left(-\mu X_{t h}\right)+\frac{\exp \left(-\mu D l_{p}\right)}{1-\exp \left(-\mu l_{p}\right)}
$$

Now let Y be the length of a randomly selected packet (with or without segmentation).

Packet length allocation is:

Published By:

Blue Eyes Intelligence Engineering 
$P(Y<y)=\left[\frac{1-\exp (-\mu y)}{1-\exp \left(-\mu X_{\text {th }}\right)}\right] P_{A}+\left[\frac{1-\exp (-\mu y)}{1-\exp \left(-\mu l_{p}\right)}\right] P_{B} \quad 0 \leq y P_{A}=\frac{E[N \mid A] P(A)}{E[N]}$

$p\left(l_{p}<Y<y\right)=\left[\frac{\exp \left(-\mu l_{p}\right)-\exp (-\mu y)}{1-\exp \left(-\mu X_{t h}\right)}\right] p_{A} \quad P_{b}=\frac{\{E[N \mid B]-1\} P(B)}{E[N]}$

$$
l_{p}<y \leq X_{t h}
$$

$p\left(Y=l_{p}\right)=p_{b}$

$$
p\left(y \leq X_{t h}\right)=1
$$

Where the likelihood of a non-segmented message packet is that a packet has a continuous length and a possibility its packet belongs to segments and a distance lower than that of a packet. The probability is [4]
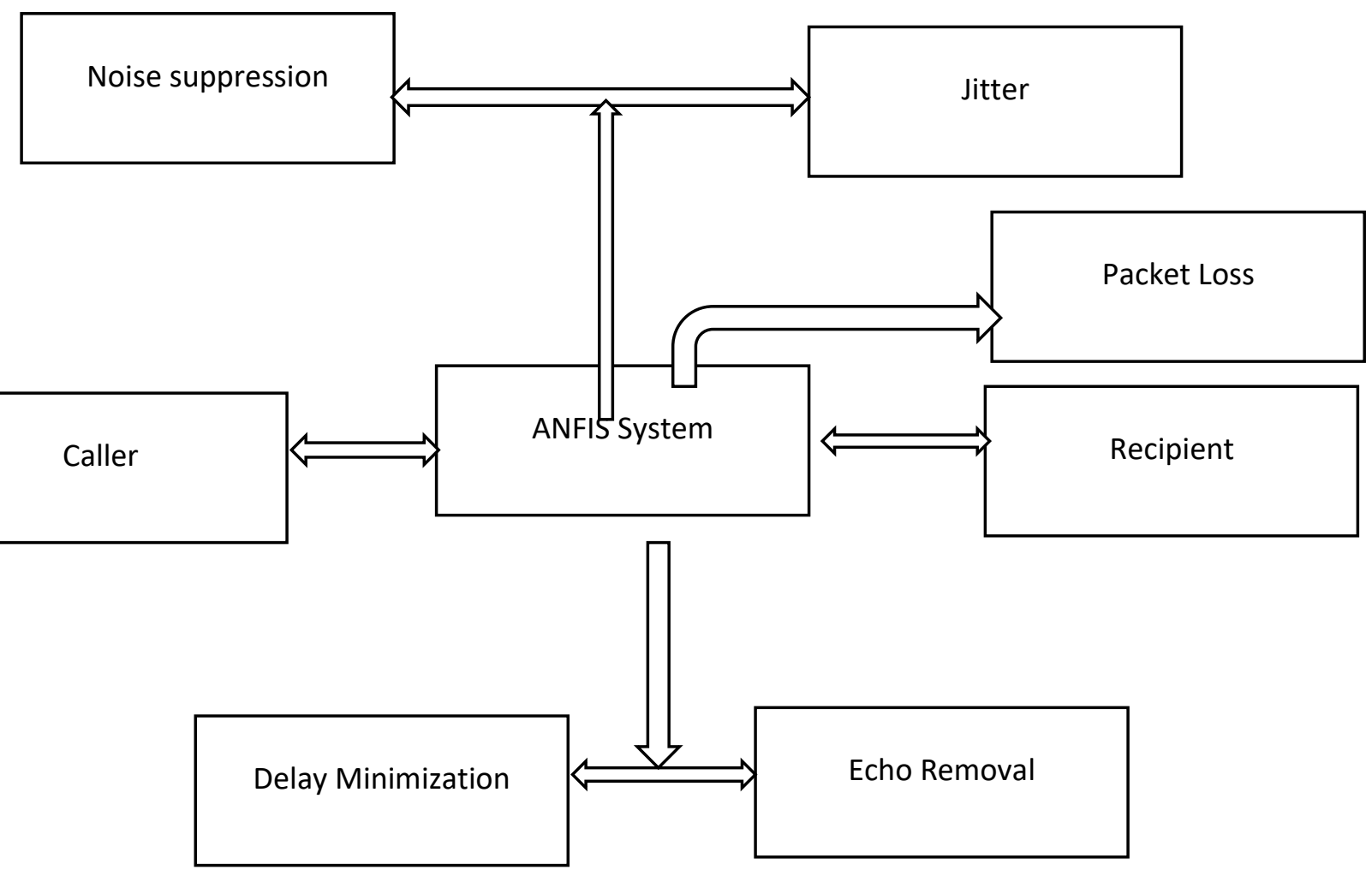

Fig 1 ANFIS Expanded System. (Source: Authors Field work) 
As shown in Fig 1 the ANFIS system which is the processing box, removes jitter, noise, minimizes delay and suppresses noise and thereby facilitates sound quality of the system.

\section{IV.RESULT AND DISCUSSION}

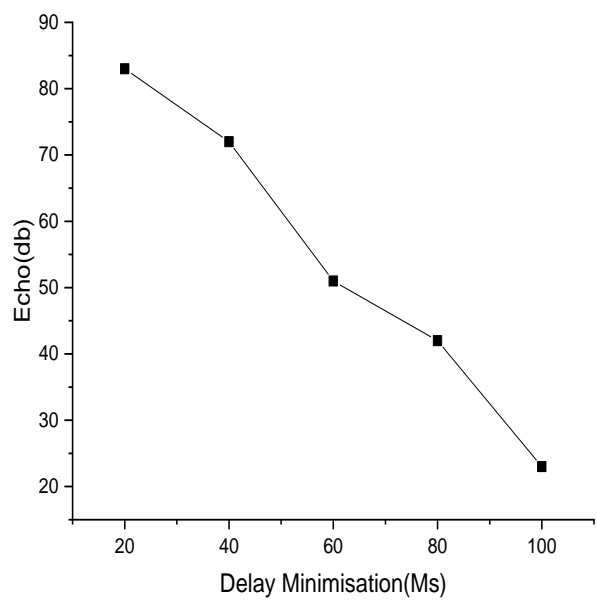

Fig 2 Variation of Echo with Delay

Listeners perceive distinct echoes when the time delay is relatively long (greater than $\sim 30$ milliseconds). When a time delay is short, listeners do not perceive echoes. Instead, a single "fused" sound is perceived. This makes it possible to create other types of effects like: chorus, flanger, phaser, and spectral filters.

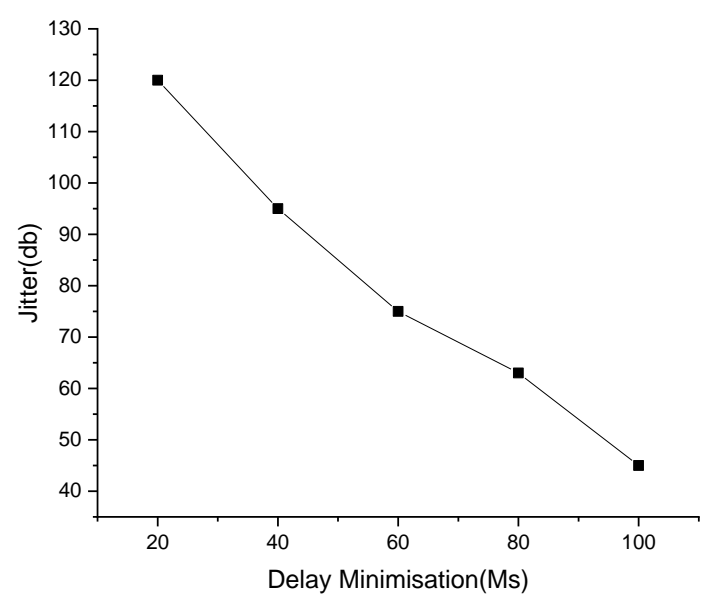

Fig 3 Effect of Jitter on Delay Minimization

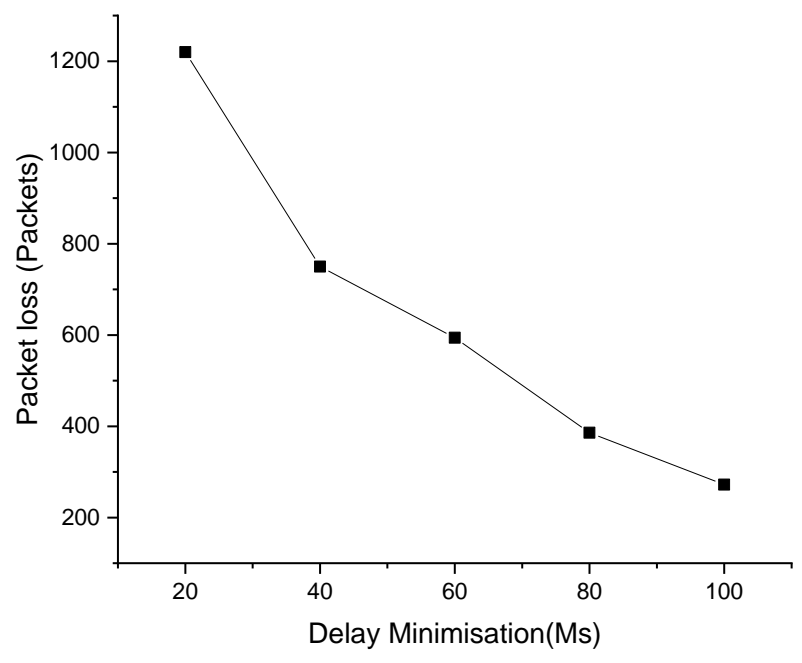

Fig 4 Variation of Packet Loss with Delay Minimization

Packet loss occurs when one or more packets of data travelling across a computer network fail to reach their destination. Packet loss is either caused by errors in data transmission, typically across wireless networks, or network congestion

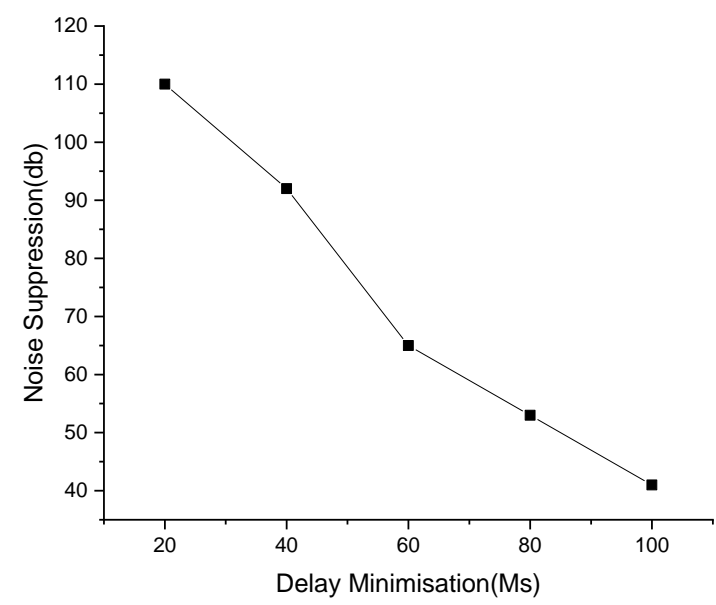

Fig 5 Variation of Noise Suppression on Delay Minimization

\section{V.CONCLUSION AND RECOMMENDATION}

The result was able to increase packet size as the delay variation reduces; the value of jitter was able to reduce noise suppression based on the echo. Better qualities both on voice quality and system performance was system. The proposed model focusses only on voice quality and will serve as an avenue to improve the institutions intranet and internet communication system Also, the results of this study can be combined with the coding techniques for future enhancement of voice quality signals.

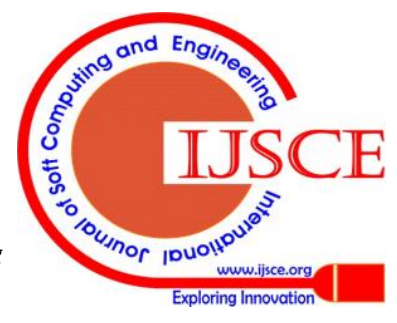




\section{REFERENCE}

1. J, Abichandani, Baenke, M, Irizarry, N, Saxena, N., P., Vyas, and Prasad, S. . Towards Layer Adaptation for Audio Transmission. International Journal of Interdisciplinary Telecommunications and Networking (IJITN)35-41., 6(4), 2014, 35-41.

2. S., Narothum, \& Y. Z., Tafesse. A Comparative Study of Voice quality and Coverage for Voice over long term Evolution calls using different codec mode-sets. IEEE Access Digital Object Identifier, 2017. Pp 675-693

3. S Jalendry, \& S. Verma. A Detailed Review on Voice over Internet Protocol (VoIP). International Journal of Engineering Trends and Technology (IJETT), 23(4), 2015. Pp 45-64.

4. H., Jung, J, Mo \& J. Park. A Data-Driven Customer Quality of Experience System for a Cellular Network. Hindawi Mobile Information $\quad$ Systems, $12 . \quad$ Retrieved from https://doi.org/10.1155/2017/4670231, 2017. Pp56-63

5. O, Slavata, \& J. Holub. Impact of the codec and various QoS methods on the final quality of the transferred voice in an IP network. Journal of Physics: Conference Series 588. doi:10.1088/17426596/588/1/012011, 2015, pp 78-88.

\section{AUTHORS PROFILE}

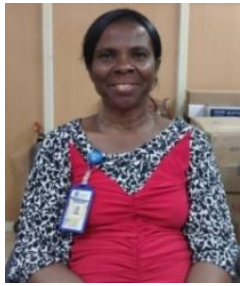

Ruth C. Amanze obtained her National Diploma (ND) in Electrical Electronics from the Federal University of Technology, Uwana, Afikpo, Ebonyi State. Her Higher National Diploma (HND) in Electronics and Telecommunication was earned from the Institute of Management and Technology (IMT), Enugu State. Her Post Graduate Diploma (PGD) and MSc. in Computer Science were earned at Babcock University, Ilishan Remo, Ogun State. She is a Research Fellow as well as staff at Babcock University. She currently serves as the Assistant Director, Department of Information Technology Development Services (ITDS). Her area of specialization is Networking and Telecommunication._amanzer@babcock.edu.ng $(+2348156414430)$

Samuel O. Okolie is a professor of Computer Science, Department of Computer Science, School of Computing and Engineering Sciences. He has been involved in coordinating the postgraduate programs for the past ten (10) years. He has expertise and research interest in Computations, Cloud Computing and Big Data Analysis. He has a wealth of industrial xperience in Computing for many years before joining academics. Official Email: kuyoroa@babcock.edu.ng Phone Number:

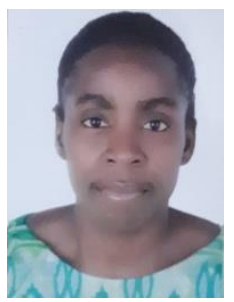

Dr. Shade Kuyoro, She is a senior lecturer at the computer Science, Babcock University. Her Research Interest is Machine Learning and Internet of Things Email: afolashadeng@gmail.com

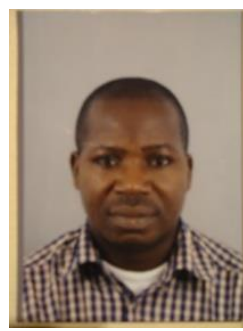

Alao Olujimi Daniel, He did his BS.c in Electrical Engineering from the University of Lagos, Nigeria, an MSc. in Computing and Information Technology from the University of Luton (UK) now Bedfordshire University Luton (UK) and a Phd in Computer Science from Babcock University, Ilishan Remo, Nigeria. He is presently a Senior lecturer in Computer Science at Babcock University Illisan- Remo. Ogun State, Nigeria. His research area are Human Computer Interaction, Computer Hardware, Networking and Telecommunication

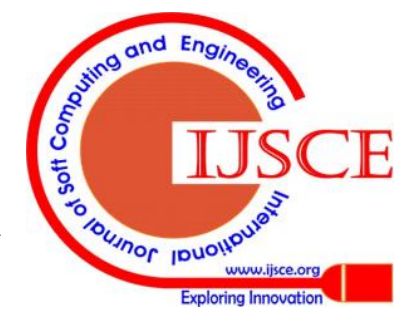

